\title{
Strategies for in-orbit calibration of drag-free control systems
}

\author{
Michel Silas Guilherme ${ }^{\mathrm{a}, *}$, Waldemar Castro Leite Filho ${ }^{\mathrm{b}}$, Stephan Theil ${ }^{\mathrm{a}}$ \\ ${ }^{a}$ ZARM/University of Bremen, Bremen, Germany \\ ${ }^{\mathrm{b}}$ IAE/CTA, Sao Jose dos Campos, Brazil \\ Received 30 August 2007; accepted 6 September 2007
}

Available online 8 September 2007

\begin{abstract}
Drag-Free Satellites (DFS) are a class of scientific satellite missions designed for research on fundamental physics as well as geodesy. They consist, basically, of a small inner satellite (test mass) located in a cavity inside a larger satellite, the normal one. The Drag-Free Attitude Control System (DFACS) is the most complex technology on-board these satellites. This key technology allows the residual accelerations on experiments on board the satellites to be significantly reduced. In order to achieve this very low disturbance environment (for some missions $<10^{-14} \mathrm{~g}$ ) the drag-free control system has to be optimized. This optimization process is required because of uncertainties in system parameters that demand a robustness of the control system. This paper will present approaches for in-orbit calibration of drag-free control systems. The discussion includes modeling, with scale factors and cross couplings, possible excitation signals, comparison of different parameter identification/estimation methods as well as simulation results.
\end{abstract}

(C) 2007 Elsevier Masson SAS. All rights reserved.

Keywords: Drag-Free Satellite; Parameter estimation; Closed-loop identification

\section{Introduction}

Drag-Free Satellites (DFS) are basically scientific satellite missions for research on fundamental physics as well as geodesy. Examples of fundamental physics missions are MICROSCOPE [15] and STEP [13,19], to test the Equivalence Principle, LISA (LISA Pathfinder) $[2,3,6]$ to detect gravitational waves and ASTROD (ASTROD I) to test relativistic gravity and to measure solar-system parameters. LISA Pathfinder will be a technological demonstrator for LISA and will test stateof-the-art sensors and actuators as well as the very complex Drag-Free Attitude Control System (DFACS) [3]. ASTROD I will also be a technological demonstrator for ASTROD. For geodesy there are missions like GRACE, successfully launched in 2002, and GOCE to be launched in the near future. Particularly for missions on fundamental physics, the DFACS plays a very important role. The disturbance reduction system must

\footnotetext{
* Corresponding author. Tel.: +49 421218 4786; fax: +49 4212184356.

E-mail addresses: michel@zarm.uni-bremen.de (M.S. Guilherme), waldemar@iae.cta.br (W.C. Leite Filho), theil@zarm.uni-bremen.de (S. Theil).
}

achieve a very low disturbance environment (for some missions $<10^{-14} \mathrm{~g}$ ). For this reason, the DFACS must be as accurate as possible.

The classical Drag-Free Satellite can be seen as two satellites in one. A small inner satellite (test mass) is located in a cavity inside of a larger (normal) satellite. The cavity contains sensors (capacitive, magnetic or optical) which determine the position of the test mass with respect to the outer satellite. The main satellite has small thrusters that provide a fine tuned thrust in order to chase the test mass, which then always remains centered in the cavity. The new generation of drag-free satellites are more complex and the satellites will have at least two test masses. New thrusters with very small thrust level, e.g. FEEP actuators [12], and new sensors will be used. The DFACS will also be more complex.

The Drag-Free Control (DFC) concept follows the approach to create a real free-fall environment by compensating the external nongravitational (nonconservative) forces on the satellite. This can be achieved either by forcing the satellite to follow a free-flying test mass or by using the test mass as an accelerometer. We can classify them in two modes of operation. "Accelerometer Mode", when the test mass works as an ac- 
celerometer, and "Displacement Mode" when the whole system works as an accelerometer.

In the Accelerometer Mode the test mass motion with respect to the satellite is driven to zero. The acceleration needed to keep the test mass at zero position is then used as a measurement for compensating the external forces. Achieving this, accelerations on the test mass as well as on the satellite can be reduced to a very low level which allows the execution of highly sensitive experiments. In the Displacement Mode the satellite is controlled to follow the test mass. The relative displacement between the test mass and satellite is measured by Electrostatic Position Suspension system, by Optical Measurement or by SQUID sensors. The kind of sensor to be used is mission dependent, but the EPS system will be always present.

The GRACE satellite, for example, had inertial sensors, for which ONERA was responsible, in accelerometer mode, as will GOCE and MICROSCOPE. STEP will also be operated with an inertial sensor in accelerometer mode, which will be the responsibility of ONERA and Stanford University, but it will have a special sensor (SQUID) to measure the difference between the test masses displacement. The LTP (LISA Technology Package) will use its inertial sensors operating in the Displacement Mode and an optical readout sensor to measure the 6 degree of freedom (6DOF) difference between the test masses. A 6DOF measurement in the Accelerometer Mode is for this mission also foreseen. Since the measurement of the test mass position cannot be obtained without applying a force on the test mass, a dynamic coupling exists between the test mass and the satellite. This coupling can be modeled as spring/damper between the two corresponding bodies (see Fig. 1). Also, negative parasitic stiffness arises due to gravitational attraction between the test mass and the satellite. The damping term can be neglected for some missions as well as the coupling between the test masses. However their estimation does not add any effort to the parameter estimation process. Beyond this linear electrostatic coupling there is also nonlinear electrostatic cross-coupling (see Fig. 2) between the different degrees of freedom, due to rotation of the test mass, and cross-talk, due to actuation in one degree of freedom that also results in actuation in other degrees of freedom. Also it is very important to know these parameters for the feedback drag-free attitude control system. Contrary of those of the linear coupling, the cross-coupling parameters can only be very well calibrated in space. The parameters above mentioned, along with the actuation gains, satellite thrusters gains and electrostatic actuation gains, make the set of parameters to be estimated in-orbit. All of these parameters must be very well known in orbit in order to improve the DFACS performance, i.e. for robustness of the controller. In a top level acceleration noise budget presented in [3], stiffness coupling and electrostatic cross-talk actuation have a major contribution. In the Accelerometer Mode, as the test mass is controlled by an internal loop in high bandwidth closed loop gain, the dynamics parameters, i.e. stiffness and possible damping, are not a source of concern. Calibrating them on the ground is sufficient for the design and performance of the internal control loop. The only calibration requirement is the general calibration process typical for almost all sensors, namely for bias, scale factors and

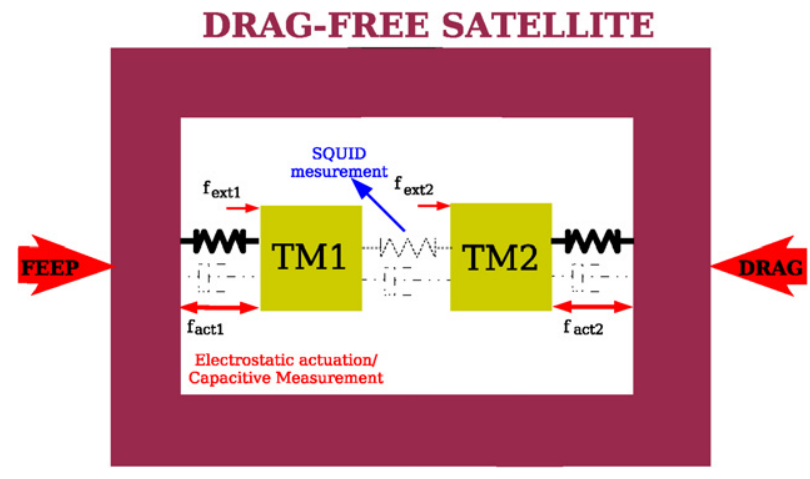

Fig. 1. Pictorial representation of a drag-free satellite with two test masses.

quadratic terms, which are especially important for missions such as MICROSCOPE, STEP and GOCE.

In the Displacement Mode usually only one test mass has high bandwidth control gain, the drag-free control bandwidth. The existence of a second test mass, which does not pursue the same center of mass as the first one, usually does not have high bandwidth control gain. In fact, as mentioned in [3], its bandwidth will be around its natural frequency. This issue, in particular, makes the coupling parameters of the second test mass critical parameters for the drag-free controller design. The more accurately they can be known the better the drag-free control system performance will be.

Beyond the reason to estimate the parameters in orbit for DFACS improvement, they are also important for the experiment measurement equation [2,3]. In the following we reproduce a measurement equation published in [2] to better explain how uncertainty in estimating the stiffness parameters affects the measurement equation. The difference (optical readout) between two test masses is described as,

$$
\begin{aligned}
\Delta x_{\text {opt }}= & \frac{1}{\omega^{2}-\left(\omega_{2 p}^{2}+\omega_{E S}^{2}\right)}\left[\frac{f_{x 2}-f_{x 1}}{m}\right. \\
& +\left(x_{1 n}+\frac{F_{s t r}}{M \omega_{D F}^{2}}\right)\left[\omega_{1 p}^{2}-\omega_{2 p}^{2}\right] \\
& \left.-\delta x \omega_{2 p}^{2}+x_{n, o p t}\left(\omega^{2}-\omega_{2 p}^{2}\right)\right]
\end{aligned}
$$

where $\Delta x_{\text {opt }}$ is the optical laser measurement between the two test masses, $x_{n, o p t}$ is the additive optical measurement noise, $\delta x$ accounts for a possible distortion of the baseline separating the two test masses, $\omega_{i p}^{2}$ is the coupling stiffness of test mass $i, m$ is the mass of the test mass (assumed to be equal for both), $\omega_{E S}^{2}$ is the electrostatic actuation on test mass 2 (TM2), $x_{1 n}$ is the EPS measurement noise of test mass 1 position with respect to the satellite, $\frac{F_{s t r}}{M \omega_{D F}^{2}}$ the satellite jitter, and $f_{i}$ are stray forces on the test mass $i$.

As one can see, the relative position noise of test mass 1 (TM1) with respect to the satellite couples to the measurement signal through $\left(\omega_{1 p}^{2}-\omega_{2 p}^{2}\right)$. As the EPS measurement is less accurate than the optical one, any mismatch in estimating the stiffness parameters (they should be equal), affects the measured experiment signal directly. 
Errors in measuring the coupling parameters for missions such as STEP and MICROSCOPE directly affect the differential measurement equation through the common mode. Assuming the coupling parameters, stiffness + damping, are known within some factor $(\Delta k, \Delta \beta)$,

$$
\begin{aligned}
\frac{k_{1}}{m_{1}} & =\frac{k}{m}-\Delta k, & \frac{k_{2}}{m_{2}} & =\frac{k}{m}-\Delta k, \\
\frac{\beta_{1}}{m_{1}} & =\frac{\beta}{m}-\Delta \beta, & \frac{\beta_{2}}{m_{2}} & =\frac{\beta}{m}-\Delta \beta,
\end{aligned}
$$

the differential mode can be described as (this equation will also be derived later in the paper)

$$
\begin{aligned}
\ddot{x}_{d} & +\left(\frac{\beta_{3}}{m_{2}}+\frac{\beta_{3}}{m_{1}}+\frac{\beta}{m}\right) \dot{x}_{d}+\left(\frac{k_{3}}{m_{2}}+\frac{k}{m_{1}}+\frac{k}{m}\right) x_{d} \\
& =-\Delta k x_{c}-\Delta \beta \dot{x}_{c}+\frac{f_{d 2}-f_{d 1}}{2}
\end{aligned}
$$

where $x_{d}$ stands for differential mode and $x_{c}$ for common mode. $k_{3}$ and $\beta_{3}$ are coupling between the two test masses and $m_{i}$ is the mass of test mass $i$. The differential mode is clearly affected by the common mode motion due to the parameter matching error. Since the common mode is controlled by the drag-free controller, the mismatch is one source of concern for drag-free control requirements. Considering all the effects, the coupling between the differential mode and common mode is conservatively assumed to be about $1 \times 10^{-4}[8,18]$. It is not a large source of concern for the measurement equation if the common mode is well controlled and the parameters well calibrated. However, an in-orbit parameter estimation process can improve the knowledge of the parameter mismatch.

\section{Electrostatic cross-coupling}

The test mass is suspended by electrostatic forces and torques, through electrodes symmetrically placed around it, which allows for the measurement of the test mass position and actuation on it.

The electrostatic interaction model equations between the test mass and the Housing Frame (HF) is nonlinear but a linearization can be carried out around small angle rotations, which permits the electrostatic interaction to be modeled as a spring-damping system, namely as

$$
\begin{aligned}
\mathbf{F}_{\text {coup }} & =\mathbf{F}_{D C}+[\mathbf{K T}] \mathbf{X}+[\mathbf{D T}] \dot{\mathbf{X}}, \\
\mathbf{T}_{\text {coup }} & =\mathbf{T}_{D C}+[\mathbf{K R}] \Omega+[\mathbf{D R}] \dot{\Omega}
\end{aligned}
$$

where $F_{D C}$ and $T_{D C}$ are the $D C$ forces and torques, respectively, $\mathbf{X}$ is the 3 DOF translational motion, $\Omega$ is the 3 DOF attitude of the test mass, and ([KT], $[\mathbf{D T}])$ and ([KR], [DR]) account for the rotational and translational coupling coefficients, respectively. Due to rotation of the test mass (see Fig. 2), there will be cross-coupling interaction between different degrees of freedom [4]. Again, in a linearized form, the equation can now be modeled as

$$
\begin{aligned}
& \mathbf{F}_{\text {coup }}=\mathbf{F}_{D C}+[\mathbf{K T}] \mathbf{X}+[\mathbf{D T}] \dot{\mathbf{X}}+[\mathbf{K T R}] \Omega, \\
& \mathbf{T}_{\text {coup }}=\mathbf{T}_{D C}+[\mathbf{K R}] \Omega+[\mathbf{D R}] \dot{\Omega}+[\mathbf{K R T}] \mathbf{X}
\end{aligned}
$$
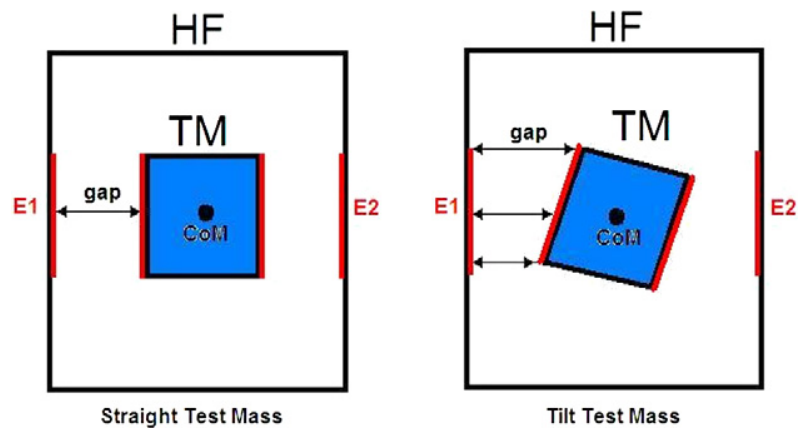

Fig. 2. On the left side a straight TM and on the right side a tilt TM.

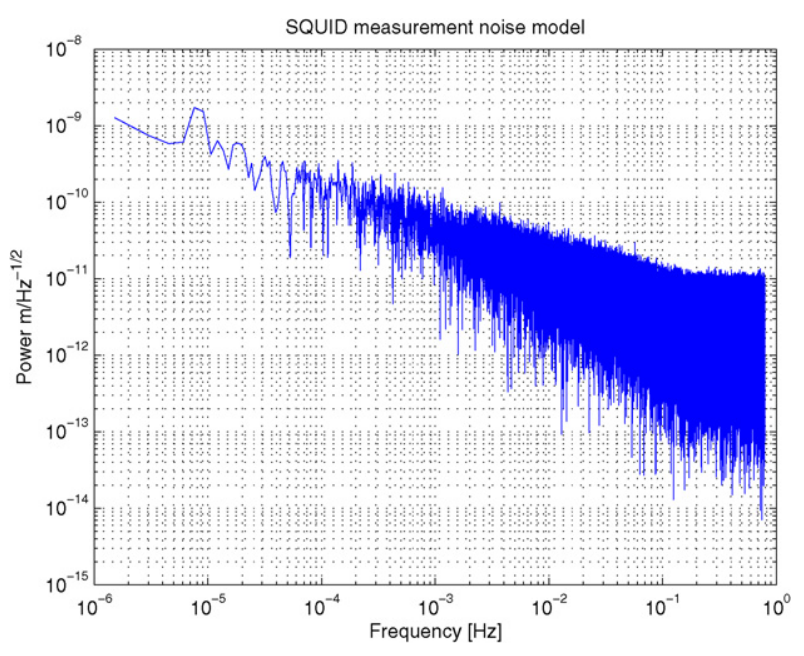

Fig. 3. Frequency dependent SQUID noise measurement.

where the added terms KTR and KRT account for the crosscoupling (or cross-talk in the case of actuation) coefficients, i.e. coupling between the translation and attitude of the test mass.

\section{Measurement noise models}

The SQUID sensor is intended to be used in the STEP mission $[18,19]$. This is a very accurate sensor and the measurement accuracy is frequency dependent, showing a $1 / f$ characteristic (see Fig. 3). The electrostatic suspension measurement system measures six degrees of freedom as well as actuates on the test mass positions $[2,3,20]$. The displacement accuracy is also frequency dependent (see Fig. 4). This electrostatic measurement will be present in all drag-free missions. The optical measurement, or laser readout $[2,3]$, is a very accurate measurement, i.e. its noise level is very small in the frequency measurement bandwidth. This sensor is intended to be used for LISA/Lisa Pathfinder (see Fig. 5).

\section{Accelerometer calibration}

The acceleration signal is mainly important for missions to test the Equivalence Principle and for Geodesy. For the first purpose, as the experiment signals are directly extracted from the acceleration measurements, there will be a very high performance requirement upon the accelerometer. 


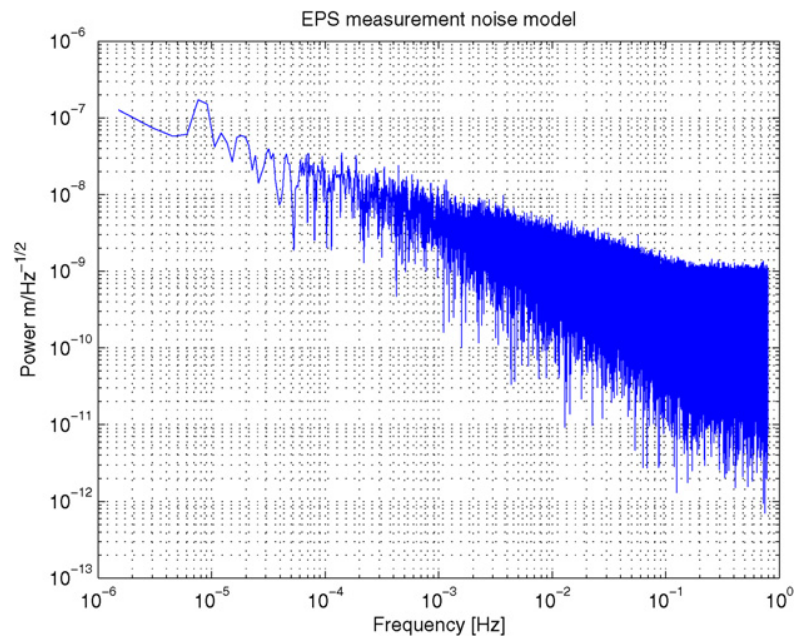

Fig. 4. Frequency dependent EPS noise measurement.

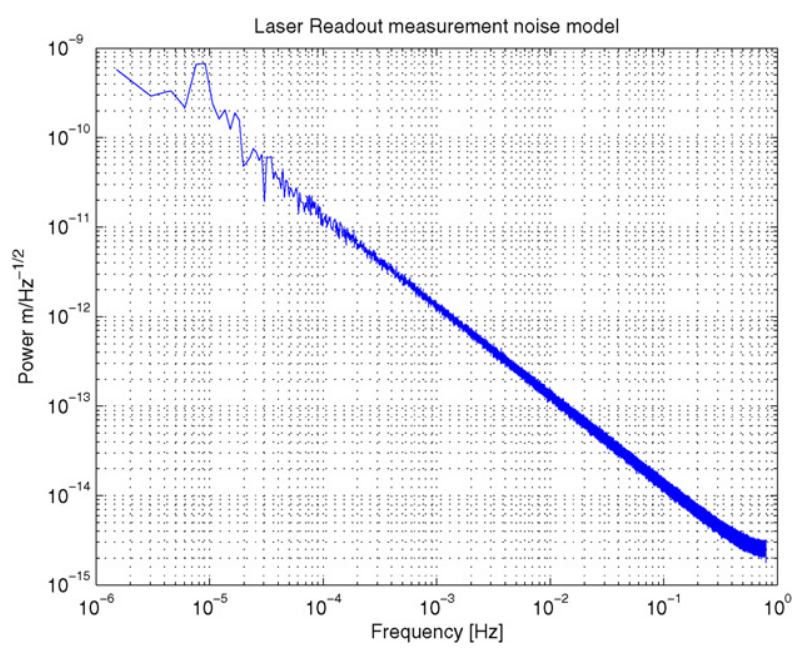

Fig. 5. Frequency dependent laser noise readout.

Regarding perturbation studies from previous missions, the scientific measurements made on a Drag-Free mission might be corrupted by intrinsic defects of the instrument. Although the amplitudes of the disturbing forces (nongravitational, Earth's gravity gradient) are reduced by the DFACS, a calibration procedure for the instrument is necessary to measure the amplitudes of these defects and correct the scientific measurements by an a posteriori data treatment [15].

Let us consider the following linear model of acceleration's measurement $(\Gamma)$ of one inertial sensor in three degrees of freedom as

$\Gamma_{\text {measured }}=K_{0}+M \cdot \Gamma_{\text {excitation }}+\Gamma_{\text {noise }}$

where $K_{0}$ is the intrinsic bias of the instrument due to cage dis-symmetries and potential offsets, $M$ is the instrument sensitivity matrix and $\Gamma_{\text {noise }}$ the intrinsic instrument acceleration noise (around $3.8 \times 10^{-12} \mathrm{~m} / \mathrm{s}^{2} / \sqrt{\mathrm{Hz}}$ according to [15]). The sensitivity matrix $M$, besides the scale factors of the instrument along the three axes, includes the coupling elements between the instrument axis, induced by the perpendicularity defects of the test mass.
The instrument in-orbit acceleration calibration consists in estimating the matrix $M^{-1}$ in order to correct the measurement acceleration, and so obtain the true acceleration. This work has been done and presented in [5].

\section{Equations of motion}

The following derivation of the models is based on the work published in $[8,10,19]$. In this paper the equations will be developed considering two test masses only. However, expansion to more than two test masses is straightforward. The plant will be split in translation equations of motion and attitude. Then we have,

\section{- Translation}

$$
\begin{aligned}
M \ddot{\mathbf{X}}_{s}= & \mathbf{F}_{F E E P}+\mathbf{F}_{\text {DRAG }}-\mathbf{F}_{\text {act } 1}-\mathbf{F}_{a c t 2} \\
& -\mathbf{F}_{\text {coup } 1}-\mathbf{F}_{\text {coup } 2} \\
m_{1} \ddot{\mathbf{X}}_{i}= & \mathbf{F}_{\text {acti }}-\mathbf{F}_{\text {coup } i}-\mathbf{F}_{\text {coup } 1 / 2 i}+\mathbf{F}_{\text {PERTi }}
\end{aligned}
$$

where $\mathrm{X}_{i}$ is the absolute position of test mass $i, m_{i}$ and $M$ are the test mass $i$ and satellite masses, respectively, $\mathbf{F}_{F E E P}$ is the FEEP actuation thruster, $\mathbf{F}_{D R A G}$ the overall disturbance on the satellite, $\mathbf{F}_{a c t i}$ is the electrostatic actuation forces on test mass $i$, $\mathbf{F}_{\text {coupi }}$ the coupling between the test mass $i$ and the satellite and $\mathbf{F}_{\text {PERTi }}$ the perturbation on the test mass $i$. The term $\mathbf{F}_{\text {coup } 1 / 2 i}$ accounts for a possible coupling between the test masses.

Then the test masses equations are described as,

$m_{1} \ddot{\mathbf{X}}_{1}=\mathbf{F}_{\text {act } 1}-\mathbf{F}_{\text {coup } 1}-\mathbf{F}_{\text {coup } 1 / 2}+\mathbf{F}_{\text {PERT } 1}$,

$m_{2} \ddot{\mathbf{X}}_{2}=\mathbf{F}_{\text {act } 2}-\mathbf{F}_{\text {coup } 2}+\mathbf{F}_{\text {coup } 1 / 2}+\mathbf{F}_{\text {PERT } 2}$.

Substituting

$\mathbf{X}_{1}=\mathbf{x}_{1}+\mathbf{X}_{s} ; \quad \mathbf{X}_{2}=\mathbf{x}_{2}+\mathbf{X}_{s}$

in Eq. (6), and neglecting perturbation on the test mass, we have

$\ddot{\mathbf{x}}_{1}=\frac{\mathbf{F}_{\text {act } 1}}{m_{1}}-\frac{\mathbf{F}_{\text {coup } 1}}{m_{1}}-\frac{\mathbf{F}_{\text {coup } 1 / 2}}{m_{1}}-\ddot{\mathbf{X}}_{s}$,

$\ddot{\mathbf{x}}_{2}=\frac{\mathbf{F}_{\text {act } 2}}{m_{2}}-\frac{\mathbf{F}_{\text {coup } 2}}{m_{2}}+\frac{\mathbf{F}_{\text {coup } 1 / 2}}{m_{2}}-\ddot{\mathbf{X}}_{s}$

where $\mathbf{x}_{1}$ is the relative displacement between the test mass and the satellite, or between the test mass and the HF.

\section{- Attitude}

Considering small angle rotations the satellite and test mass attitude can be described, in a linearized compact form [21], as $[I]_{i} \ddot{\Omega}_{i}=\mathbf{T}_{\text {exti }}$

where $\mathbf{I}_{i}$ is the body $i$ inertia matrix, $\ddot{\Omega}_{i}$ is the attitude of body $i$ and $\mathbf{T}_{\text {exti }}$ the external torque acting on the body $i$.

Furthermore, the external torque can be modeled as

$\mathbf{T}_{\text {exti }}=\mathbf{T}_{\text {acti }}+\mathbf{T}_{\text {coupi }}+\mathbf{T}_{\text {PERTi }}$.

For the electrostatic attitude coupling presented in Section 2 and neglecting the perturbation on the test mass, the test mass attitude equation results in

$$
\begin{aligned}
{[I]_{i} \ddot{\Omega}_{i}=} & \mathbf{T}_{a c t i}-\mathbf{T}_{D C i}-[\mathbf{K R}] \Omega_{i}-[\mathbf{D R}] \dot{\Omega}_{i} \\
& -[\mathbf{K R T}] \mathbf{X}_{i} .
\end{aligned}
$$




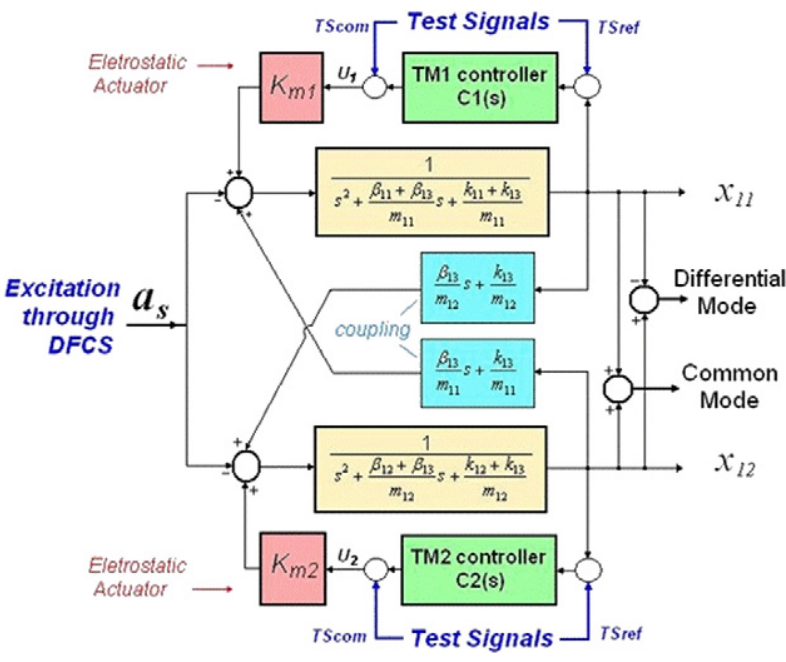

Fig. 6. Accelerometer block diagram.

The test mass attitude equations can be put in a state-space form as

$\underline{\dot{\Omega}}_{i}=\left[\begin{array}{cc}\mathbf{0} & \mathbf{I} \\ -\frac{[K R] i}{I_{i}} & -\frac{[D R] i}{I_{i}}\end{array}\right] \underline{\Omega}_{i}+\left[\begin{array}{cc}\mathbf{0} & \mathbf{0} \\ \underline{g}^{T} & {[K R T]_{i}}\end{array}\right]\left[\begin{array}{c}\underline{T}_{a c t i} \\ \underline{x}_{i}\end{array}\right]$,

$\underline{\Omega}_{\text {out }}=\left[\begin{array}{ll}\mathbf{I} & \mathbf{0}\end{array}\right] \underline{\Omega}_{i}$

where $\mathbf{I}$ is the identity matrix, $\mathbf{0}$ is a null matrix and $\underline{g}^{T}$ is the torque actuation gain.

\section{Drag-Free control system}

In the Accelerometer Mode (see Fig. 6) the test masses are controlled by an internal loop in high bandwidth control gain. It is assumed that the control loop feeds back the test mass position. Calling the controller for test mass $1 C_{1}(s)$ and for test mass $2 C_{2}(s)$, and assuming them to be identical, the actuation for each test mass can be written as

$$
\begin{aligned}
\frac{F_{a c t i}}{m_{i j}}(s) & =K_{m i}(s) \cdot U_{i}(s) \\
& =K_{m i}(s) \cdot\left[T S_{\text {icom }}+C_{j}(s) \cdot\left(T S_{\text {iref }}-x_{i}(s)\right)\right]
\end{aligned}
$$

where $K_{m i}(s)$ is the test mass actuation gain.

As described in [2,3], some degrees of freedom are drag-free controlled and some are controlled by the EPS system. For the degrees of freedom controlled by the EPS system, the control loop feeds back the test mass positions and the actuation equation can also be described as Eq. (13). The controllers for these degrees of freedom have low bandwidth control gain.

Regarding the identification test signals (TS), they can be placed as a reference for the control system $\left(T S_{\text {iref }}\right)$ or as a command signal to the actuators $\left(T S_{\text {icom }}\right)$, which is added to the output of the controller (see Fig. 6). In the first case the "Equivalent System", i.e. the plant + controller transfer function parameters, will be estimated, in the second case only the plant parameters will be estimated. Defining the parameters of coupling matrices as,

$K T(j)_{i} \equiv k_{j i}$,

$D T(j)_{i} \equiv \beta_{j i}$,
$\operatorname{KTR}(j)_{i} \equiv \gamma_{j i}$

where the index $i$ stands for the test mass number and the index $j$ stands for the parameter in the matrix. For example, $k_{11}$ is the first parameter of matrix $K T$ for test mass 1 and $k_{12}$ is the first parameter of matrix $K T$ of test mass 2 . With these denominations, from Eqs. (6) and (3), the local displacement for the test mass 1 (neglecting DC terms) can be described as,

$$
\begin{aligned}
\ddot{x}_{1}+\ddot{x}_{s}= & \frac{F_{a c t_{1}}}{m_{1}}-\frac{k_{11}}{m_{1}} \cdot x_{1}-\frac{\beta_{11}}{m_{1}} \cdot \dot{x}_{1} \\
& -\frac{k_{13}}{m_{1}} \cdot\left(x_{2}-x_{1}\right)-\frac{\beta_{13}}{m_{1}} \cdot\left(\dot{x}_{2}-\dot{x}_{1}\right) \\
& -\frac{\gamma_{11}}{m_{1}} \eta_{1}-\frac{\gamma_{21}}{m_{1}} \theta_{1}-\frac{\gamma_{31}}{m_{1}} \phi_{1} .
\end{aligned}
$$

The Laplace transform of Eq. (14) is

$$
\begin{aligned}
\left(s^{2}\right. & \left.+\frac{\beta_{11}+\beta_{13}}{m_{1}} s+\frac{k_{11}+k_{13}}{m_{1}}\right) \cdot x_{1}(s) \\
= & \left(\frac{\beta_{13}}{m_{1}} s+\frac{k_{13}}{m_{1}}\right) \cdot x_{2}(s)+\frac{F_{a c t 11}}{m_{1}}(s)-a_{s}(s) \\
& -\frac{\gamma_{11}}{m_{1}} \eta_{1}(s)-\frac{\gamma_{21}}{m_{1}} \theta_{1}(s)-\frac{\gamma_{31}}{m_{1}} \phi_{1}(s)
\end{aligned}
$$

where $a_{s}(s)$ denotes the satellite acceleration $\ddot{\mathrm{x}}_{s}$.

If we substitute Eq. (13) in (15) we get, after some manipulations, the following equation for test mass 1 ,

$$
\begin{aligned}
{[s+} & \left.\frac{\beta_{11}+\beta_{13}}{m_{1}} s+\frac{k_{11}+k_{13}}{m_{1}}+K_{m 1} C_{1}(s)\right] x_{1}(s) \\
= & \left(\frac{\beta_{13}}{m_{1}} s+\frac{k_{13}}{m_{1}}\right) x_{2}(s)+K_{m 1}\left[T S_{1 \text { command }}+C_{1}(s) T S_{1 \text { ref }}\right] \\
& -a_{s}(s)-\frac{\gamma_{11}}{m_{1}} \eta_{1}(s)-\frac{\gamma_{21}}{m_{1}} \theta_{1}(s)-\frac{\gamma_{31}}{m_{1}} \phi_{1}(s)
\end{aligned}
$$

As can be seen from Eqs. (13) and (16), in the case where the excitation signal is considered as reference for the controller, the controller structure must be considered in the identification process. This will make the identification process more complex and will increase the number of parameters to be estimated. The drag-free and attitude controller is denoted as $C_{D F}(s)$. In Accelerometer Mode, in the case of STEP for example, it is considered that the control loop feeds back information from each inertial sensor in a weighted form $\mathbf{n}$ (see Fig. 7), to control the common mode of the test masses $[15,20]$. The output of each inertial sensor is the mean of its position. Each test mass is controlled by feedbacking test mass position $q_{1}$ or $q_{2}$, by acting directly on the test mass with the EPS. The block diagram shows the situation where the test masses operate in Displacement Mode. In this case the weight $\mathbf{n}$ is dropped and some degrees of freedom $q_{D F}$ are controlled by feedbacking the test mass position and forcing the satellite to follow one test mass, through FEEP actuation. Other degrees of freedom are EPS controlled.

As for the test mass control, possible Test Signals (TS) for the satellite control can be placed as reference of the control system $T S_{\text {ref }}$ or commanded $T S_{\text {com }}$ as an additional signal to the command signal (controller signal). So, the actuation for 


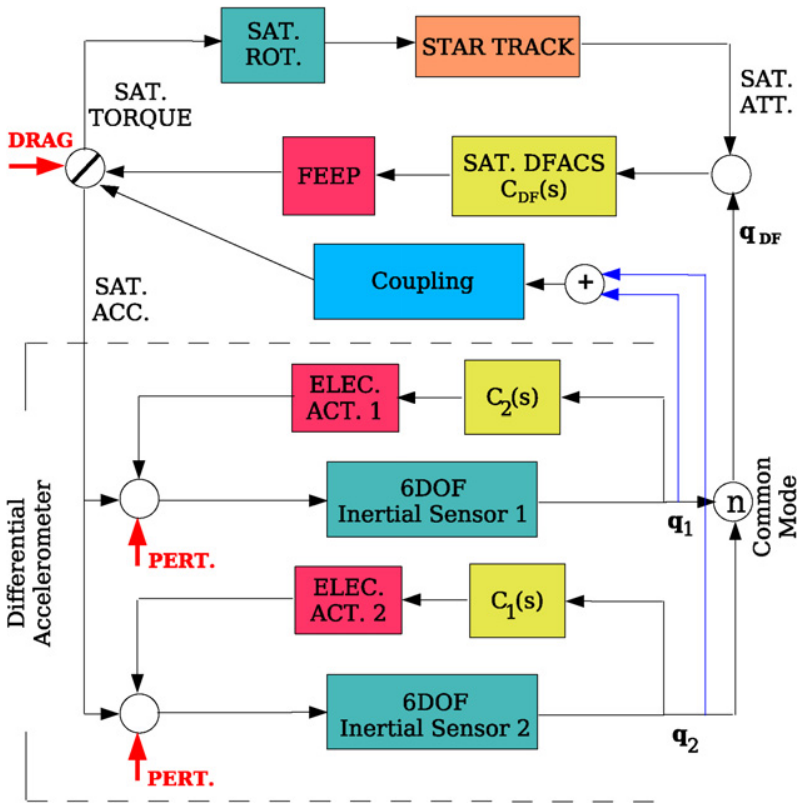

Fig. 7. Complete block diagram of a DFACS.

the satellite can be described in the same way as for the test mass, as

$$
\begin{aligned}
\frac{F_{F E E P}}{M}(s)= & K_{D F}(s) U_{D F}(s)=K_{D F}(s)\left\{T S_{D F c o m}\right. \\
& \left.+C_{D F}(s) \cdot\left[T S_{D F r e f}-\mathbf{n} \cdot \frac{x_{1}(s)+x_{2}(s)}{2}\right]\right\} .
\end{aligned}
$$

In the case of a set of coordinates, $\mathbf{q}_{d f}$, drag-free controlled (i.e. by forcing the satellite to follow one test mass position), the actuation on the satellite can be described as

$$
\begin{aligned}
\frac{F_{F E E P}}{M}(s) & =K_{D F}(s) \cdot U_{D F}(s) \\
& =K_{D F}(s) \cdot\left\{T S_{D F c o m}+C_{D F}(s) \cdot\left[T S_{D F r e f}-\mathbf{q}_{d f}\right]\right\} .
\end{aligned}
$$

\section{Experiment measurement or difference signal}

It is important for the robustness performance of the DFACS to know precisely the dynamics parameters, but it is particularly so for those of the sensitive axis. The sensitive axis is the axis that outputs the experiment signals, i.e. experiment measurement equation. It is chosen as one axis of the three orthogonal satellite axes and the displacement between the two test masses is measured by a special sensor. STEP [18] will have SQUID sensors for measuring this displacement and LISA-Pathfinder will have a Laser readout system [2,3]. The difference signal has the advantage that it is a very accurate signal and it makes it possible to cancel the satellite acceleration in the test mass equations. It must be emphasized that in the Displacement Mode the acceleration is not directly measured.

In the following we present a differential measurement equation in 3DOF. In fact, differential measurement will be done only in the sensitive axis, but statistical moments can be calculated for the difference in the other degrees of freedom, from the EPS system measurement. Choice can be made between estimating the unmeasured accelerations or estimating the difference signal in the radial axes based on statistical properties.

As stated in $[1,11,17]$, it is possible to identify the plant of each system considering it in open-loop, i.e. the controller structure does not need to be known, provided two conditions are fulfilled. Namely, that the controller structure must not be too simple one, as a proportional feedback for example, and the excitation signal must be persistently exciting (Section 9). Then, considering the difference signal as $\ddot{\mathbf{x}}_{\text {diff }} \equiv \ddot{\mathbf{x}}_{2}-\ddot{\mathbf{x}}_{1}$ and from Eq. (8), neglecting the controller, we have,

$$
\begin{aligned}
\ddot{\mathbf{x}}_{\text {diff }}= & \frac{\mathbf{F}_{a c t 2}}{m_{2}}-\frac{\mathbf{F}_{\text {act } 1}}{m_{1}}+\frac{\mathbf{F}_{\text {coup } 2}}{m_{2}}+\frac{\mathbf{F}_{\text {coup } 1}}{m_{1}} \\
& +\mathbf{F}_{\text {coup } 1 / 2}\left(\frac{m_{1}+m_{2}}{m_{1} m_{2}}\right) .
\end{aligned}
$$

Inserting the coupling force equation (Eq. (3)) in Eq. (19), results in

$$
\begin{aligned}
\ddot{\mathbf{x}}_{\text {diff }}= & \frac{\mathbf{F}_{a c t 2}}{m_{2}}-\frac{\mathbf{F}_{a c t 1}}{m_{1}}+\mathbf{F}_{\text {coup } 1 / 2}\left(\frac{m_{1}+m_{2}}{m_{1} m_{2}}\right) \\
& -\frac{\mathbf{F}_{D C 2}+[\mathbf{K T}]_{2} \mathbf{x}_{2}+[\mathbf{D T}]_{2} \dot{\mathbf{x}}_{2}+[\mathbf{K T R}]_{2} \Omega_{2}}{m_{2}} \\
& +\frac{\mathbf{F}_{D C 1}+[\mathbf{K T}]_{1} \mathbf{x}_{1}+[\mathbf{D T}]_{1} \dot{\mathbf{x}}_{1}+[\mathbf{K T R}]_{1} \Omega_{1}}{m_{1}} .
\end{aligned}
$$

Introducing $\ddot{\mathbf{x}}_{2}=\ddot{\mathbf{x}}_{\text {diff }}+\ddot{\mathbf{x}}_{1}$ in Eq. (20) we get the final differential equation as,

$$
\begin{aligned}
\ddot{\mathbf{x}}_{\text {diff }}= & -\frac{[\mathbf{K T}]_{2}}{m_{2}} \mathbf{x}_{\text {diff }}-\frac{[\mathbf{D T}]_{2}}{m_{2}} \dot{\mathbf{x}}_{\text {diff }} \\
& +\frac{\mathbf{F}_{D C 1}}{m_{1}}-\frac{\mathbf{F}_{D C 2}}{m_{2}}+\frac{\mathbf{F}_{a c t 2}}{m_{2}}-\frac{\mathbf{F}_{a c t 1}}{m_{1}} \\
& +\frac{\left([\mathbf{K T}]_{1}-[\mathbf{K T}]_{2}\right) \mathbf{x}_{1}+\left([\mathbf{D T}]_{1}-[\mathbf{D T}]_{2}\right) \dot{\mathbf{x}}_{1}}{m_{1}} \\
& +\frac{[\mathbf{K T R}]_{1} \Omega_{1}}{m_{1}}-\frac{[\mathbf{K T R}]_{2} \Omega_{2}}{m_{2}} \\
& +\mathbf{F}_{\text {coup } 1 / 2}\left(\frac{m_{1}+m_{2}}{m_{1} m_{2}}\right) .
\end{aligned}
$$

Taking the Laplace transform of Eq. (21), considering only diagonal coupling and cross-coupling, we have,

$$
\begin{aligned}
D_{1}(s) \cdot x_{\text {diff }}(s)= & N_{1}(s) \cdot x_{1}(s)-K_{m 1}(s) \cdot U_{1}(s) \\
& +K_{m 2}(s) \cdot U_{2}(s)-\frac{\gamma_{11}}{m_{1}} \eta_{1}(s) \\
& +\frac{\gamma_{12}}{m_{2}} \eta_{2}(s)
\end{aligned}
$$

where

$$
\begin{aligned}
& D_{1}(s)=s^{2}+\left(\frac{\beta_{12}+\beta_{13}}{m_{2}}+\frac{\beta_{13}}{m_{1}}\right) s+\left(\frac{k_{12}+k_{13}}{m_{2}}+\frac{k_{13}}{m_{1}}\right), \\
& N_{1}(s)=\left(\frac{\beta_{11}}{m_{1}}-\frac{\beta_{12}}{m_{2}}\right) s+\left(\frac{k_{11}}{m_{1}}-\frac{k_{12}}{m_{2}}\right) .
\end{aligned}
$$

The system has $x_{\text {diff }}$ as output and 5 inputs $U_{1}, U_{2}, x_{1}, \eta_{1}, \eta_{2}$ and the disturbance $F_{D r a g}$, as shown in Fig. 8.

In a state-space form the difference equation can be described as 


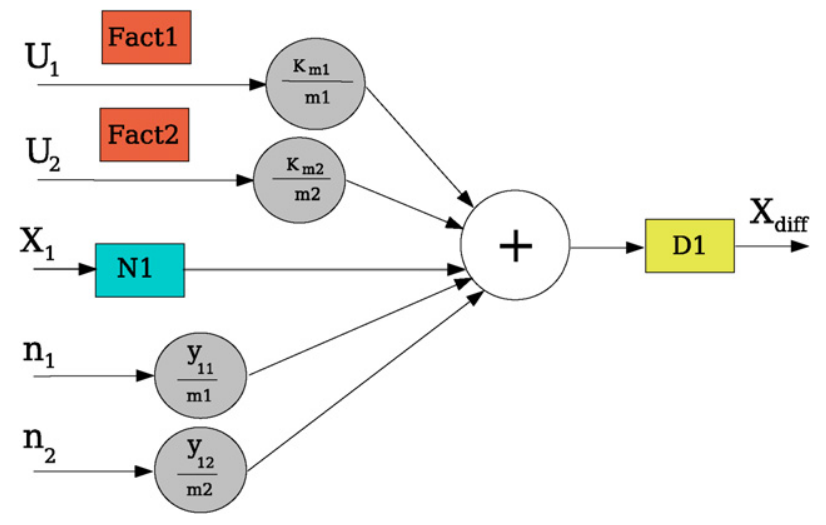

Fig. 8. Block diagram of a open-loop multi-input single-output (MISO) system for estimation of the test mass actuation gains and coupling parameters.

$$
\begin{aligned}
\underline{\dot{X}}= & {\left[\begin{array}{ccc}
0 & -\frac{k_{12}+k_{13}}{m_{2}}-\frac{k_{13}}{m_{1}} \\
1 & -\frac{\beta_{12}+\beta_{13}}{m_{2}}-\frac{\beta_{13}}{m_{1}}
\end{array}\right] \underline{X} } \\
& +\left[\begin{array}{ccccc}
K_{m 1} & K_{m 2} & \frac{k_{11}}{m_{1}}-\frac{k_{12}}{m_{2}} & -\gamma_{11} & \gamma_{12} \\
0 & 0 & \frac{\beta_{11}}{m_{1}}-\frac{\beta_{12}}{m_{2}} & 0 & 0
\end{array}\right]\left[\begin{array}{c}
U_{1} \\
U_{2} \\
x_{1} \\
\eta_{1} \\
\eta_{2}
\end{array}\right],
\end{aligned}
$$$$
x_{\text {diff }}=\left[\begin{array}{ll}
0 & 1
\end{array}\right] \underline{X} .
$$

\section{Satellite equation to estimate $K_{D F}$ and other coupling parameters}

The satellite equation of motion must be used for parameter estimation in two situations: when coupling between the test masses exists (because the physical coefficients cannot be calculated from the estimated parameters of the difference equation alone) and to estimate the FEEP gain. The equations of motion for the satellite, considering only diagonal cross-coupling, can be written as,

$$
\begin{aligned}
\ddot{\mathrm{x}}_{s}= & \frac{F_{F E E P}}{M}-\frac{F_{D r a g}}{M}-\frac{F_{a c t 1}}{M}+\frac{k_{11}}{M} \cdot x_{1} \\
& +\frac{\beta_{11}}{M} \cdot \dot{x}_{1}-\frac{F_{a c t 2}}{M}+\frac{k_{12}}{M} \cdot x_{2} \\
& +\frac{\beta_{12}}{M} \cdot \dot{x}_{2}+\frac{\gamma_{11}}{M} \eta_{1}+\frac{\gamma_{12}}{M} \eta_{2} .
\end{aligned}
$$

The Laplace transform of Eq. (24) results in

$$
\begin{aligned}
a_{s}(s) & =K_{D F}(s) U_{D F}(s)-\frac{F_{D r a g}}{M}(s) \\
& -\frac{K_{m 1}(s)}{M} U_{1}(s)+\frac{\beta_{11} s+k_{11}}{M} x_{1}(s) \\
& -\frac{K_{m 2}(s)}{M} U_{2}(s)+\frac{\beta_{12} s+k_{12}}{M} x_{2}(s) \\
& +\frac{\gamma_{11}}{M} \eta_{1}(s)+\frac{\gamma_{12}}{M} \eta_{2}(s) .
\end{aligned}
$$

Eq. (25) describes the dynamics of the controlled satellite.

When the satellite acceleration is not directly measured another output signal must be defined. Any inertial sensor position can be defined as the output. Let us consider $x_{1}$ as the output.

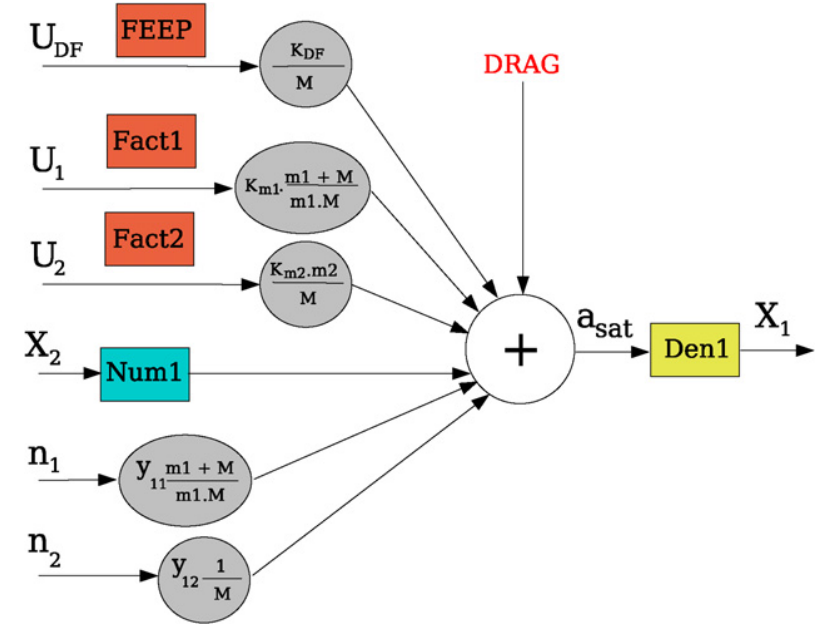

Fig. 9. Block diagram of a open-loop multi-input single-output (MISO) system for estimation of the drag-free control system gain.

Substituting Eq. (25) in Eq. (16), we get, after some manipulations,

$$
\begin{aligned}
x_{1}(s) & =\frac{1}{\operatorname{Den}_{1}(s)} \cdot\left[-K_{D F}(s) \cdot U_{D F}(s)\right. \\
& +\left(\frac{M+m_{1}}{m_{1} M}\right) K_{m 1}(s) \cdot U_{1}(s)+N u m_{1}(s) x_{2}(s) \\
& +\frac{m_{2}}{M} K_{m 2}(s) U_{2}(s)-\left(\frac{M+m_{1}}{m_{1} M}\right) \gamma_{11} \eta_{1}(s) \\
& \left.-\frac{\gamma_{12}}{M} \eta_{2}(s)+\frac{F_{D r a g}}{M}(s)\right]
\end{aligned}
$$

where

$$
\begin{aligned}
& \operatorname{Den}_{1}(s)=s^{2}+\left(\frac{\beta_{11}+\beta_{13}}{m_{1}}+\frac{\beta_{11}}{M}\right) \cdot s+\frac{k_{11}+k_{13}}{m_{1}}+\frac{k_{11}}{M}, \\
& \operatorname{Num}_{1}(s)=\left(\frac{\beta_{13}}{m_{1}}-\frac{\beta_{12}}{M}\right) \cdot s+\frac{k_{13}}{m_{1}}-\frac{k_{12}}{M} .
\end{aligned}
$$

The process has $x_{1}$ as output and 7 inputs $U_{D F}, U_{1}, U_{2}, x_{2}$, $\eta_{1}, \eta_{2}$ and the disturbance $F_{\text {Drag }}$, as shown in Fig. 9. Putting Eq. (26) into state-space formulation, results in

$$
\underline{X}=[A] \underline{X}+[B]^{T}\left[\begin{array}{c}
U_{D F} \\
U_{11} \\
U_{12} \\
x_{12} \\
\eta_{1} \\
\eta_{2} \\
F_{\text {Drag }}
\end{array}\right],
$$

where

$$
[A]=\left[\begin{array}{cc}
0 & -\frac{k_{11}+k_{13}}{m_{1}}-\frac{k_{11}}{M} \\
1 & -\frac{\beta_{11}+\beta_{13}}{m_{1}}-\frac{\beta_{11}}{M}
\end{array}\right],
$$




$$
\begin{aligned}
{[B] } & =\left[\begin{array}{cc}
-K_{D F} & 0 \\
\mu_{1} K_{m 1} & 0 \\
\frac{K_{m 2}}{M} m_{2} & 0 \\
\frac{k_{13}}{m_{1}}-\frac{k_{12}}{M} & \frac{\beta_{13}}{m_{1}}-\frac{\beta_{12}}{M} \\
-\mu_{1} \gamma_{11} & 0 \\
-\frac{\gamma_{12}}{M} & 0 \\
\frac{1}{M} & 0
\end{array}\right], \\
\mu_{1} & \equiv\left(\frac{M+m_{1}}{m_{1} M}\right) .
\end{aligned}
$$

\section{Input signals}

Following $[11,14,17]$, to estimate parameters in the closed loop it is necessary to guarantee that the input signals will be persistently excited. An input signal is persistently exciting if its covariance matrix is nonsingular [11]. Also, for each parameter to be estimated a different frequency in the spectrum is necessary. It means that, for $n$ parameters to be estimated there must be at least $n$ nonprime frequencies in the input signal. We have considered three input signals to excite the satellite and the test masses. A Sinusoidal or Multi-sines and a Chirp signal (periodic signals), and a Pseudo Random Binary Signal (PRBS). Frequencies inside and around the system bandwidth have been chosen in order to well excite the plant [16].

Sinusoidal or Multi-sines Input: Sinusoidal or Multi-Sines signal is widely used for parameter estimation, because of a direct insight of its frequency spectrum. Multi-sines signals are built when more than one parameter of a system must be estimated. It comprises a sum of sinusoidal signals with $n$ different frequencies, when $n$ parameters have to be estimated. To assure a rich spectrum, $n$ prime number multiples of a base frequency must be used, in order to avoid multiples of the same base frequency at the same position in the spectrum, and each added sinusoidal signal must have a different phase. It guarantees the signal as being of order of persistency $n$. This signal has the disadvantages that its power decreases with the number of added sines and the estimated parameters can present high correlation [9].

Chirp input: A chirp is a signal in which the frequency increases ('up-chirp') or decreases ('down-chirp') with time. It is also a periodic signal and the only disadvantage is that the signal has power outside the frequency range of interest. This can introduces frequency components with lower signal to noise ratio [16].

PRBS input: PRBS is a deterministic signal with whitenoise-like properties [11,17]. A three-levels PRBS was chosen because it can help to identify nonlinearities. The built signal has its time period divided into 19 parts. The sequences were chosen such that the mean value is zero. This signal has a whitenoise-like property. So, choosing one fundamental frequency below the lower frequency of the system bandwidth, guarantees that the system will be well excited. Because of its characteristics this signal should be the most appropriate choice. However, as the controller structure is not known, any derivative term (as a PID controller for example) would generate prohibited com- mands. Besides that, using this signal as a command (added to the controller signal) will result in practical problems because any off-set will be integrated directly by the test mass dynamics.

\section{Parameter estimation methods}

The system model can be described as transfer functions or state-space models, as developed before. Transfer functions, or rational models, such as ARX and ARMAX can be used and the difference between them is how the noise enters the system. Some representation for nonlinear system such as NARMAX is also possible, but the model is more complex [11,17]. On the other hand, state-space models give more flexibility in describing a system, be it linear or nonlinear, for example in modeling state and measurement bias [7].

Having chosen one structure, the second step is to choose the parameter estimation method. They can be Least Squares Estimation (LSE), Maximum Likelihood + Output Error Method (MLE + OEM), in which the cost function optimization is ponderated by a Maximum Likelihood information of the residuals, Extended Kalman Filter and Instrument Variables [7,11]. The set of parameter estimation methods mentioned above is not exhaustive. Which one will be used depends on the model structure and on how the noise enters the system. In the following we will denote MLE + OEM by OEM only.

In this work we used the state-space model when using the OEM method and transfer functions when using the LSE method. The reason to use transfer functions when estimating with LSE is that for the state space application the states must also be available. LSE is a one-shot method, i.e. noniterative, and OEM an iterative method (see Fig. 10).

We have built two versions of the LSE, namely the Ordinary Least Squares (OLS) and the Total Least Squares (TLS). The LSE is the normal approach shown in many text books about parameter estimation. Starting from an ARX model, for example, we have the following linear equation

$Y=\Theta X+\varepsilon$

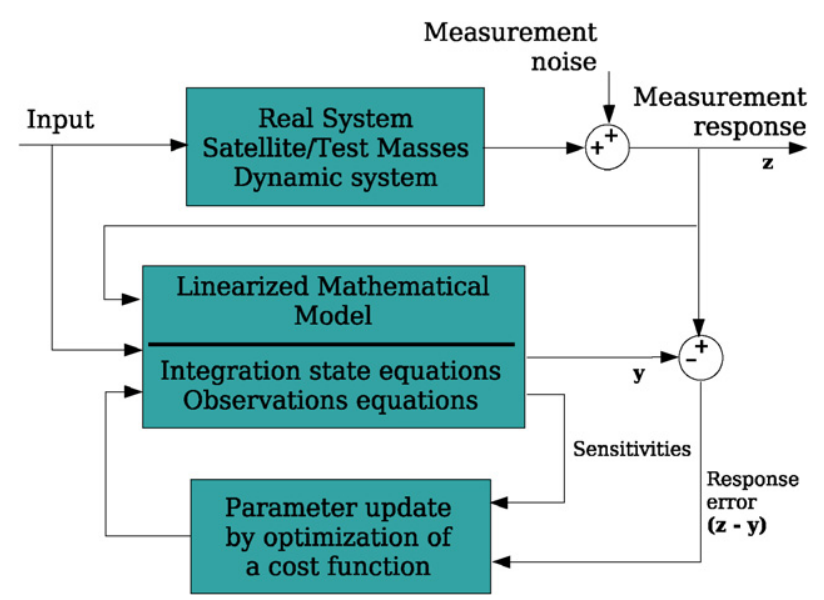

Fig. 10. Block schematic of output error parameter estimation method applied for a drag-free control system. 
where $\varepsilon$ is the prediction error, $X$ the regression matrix, or independent variables, and $Y$ the dependent variables, or output. Then, the OLS method, from a quadratic cost function, is described as

$\hat{\Theta}_{O L S}=\left(X^{T} X\right)^{-1} X^{T} Y$

in the case where the number of observations is larger than the number of parameters to be estimated.

The TLS method is an adaptation from the LSE and accounts for noise in the inputs [7]. The method can be described as follows. Starting from the same regression equation we add a term $\mu$ to account for noise in the regressors as

$Y=(X-\mu) \Theta+\varepsilon$.

Based on singular value decomposition, the estimated parameters are

$\hat{\Theta}_{T L S}=\left(X^{T} X-\sigma_{n+1}^{2} \mathbf{I}\right)^{-1} X^{T} Y$

where $\sigma_{n+1}^{2}$ is the smallest singular value of the compounded matrix $\left[\begin{array}{ll}X & Y\end{array}\right]$, I the identity matrix and $n$ the output dimension.

It must be emphasized that both methods (OLS and TLS) yields biased estimates in the presence of systematic errors, e.g. input bias.

In general, a system can be described in state-space form as

$\dot{x}=A x+B u+b_{x}+F w$,

$y=C x+b_{y}$,

$z=y+G v+b_{z}$

where $b_{x}=A x_{0}-B \Delta u$ and $b_{y}=C x_{0}+\Delta u z$, are the lumped bias parameters and $b_{z}$ the measurement bias. The lumped bias parameters account for DC bias, initial conditions and variation in the control input. $F$ is the possible process noise matrix and $G$ the measurement noise matrix.

The OEM can be described as following. Based on stochastic properties a cost function is developed

$$
\begin{aligned}
J(\Theta, R)= & \frac{1}{2} \sum_{k=1}^{N}[z(k)-y(k)]^{T} R^{-1}[z(k)-y(k)] \\
& +\frac{N}{2} \ln [\operatorname{det}(R)]+\frac{N n_{y}}{2} \ln (2 \pi)
\end{aligned}
$$

where $\Theta$ is the parameter vector, to be estimated, $z(k)$ the measurement and $y(k)$ the simulated output at time $t_{k}$, respectively. $R$ the measurement error covariance matrix, $N$ the data length and $n_{y}$ the output dimension.

In the case of the unknown measurement noise covariance matrix, we can take the maximum likelihood estimate of $R$ as

$R=\frac{1}{N} \sum_{k=1}^{N}[z(k)-y(k)][z(k)-y(k)]^{T}$.

In doing so, the covariance matrix becomes constant. Considering also that $n_{y}$ and $N$ are fixed for a postulated model and data set being analyzed, the cost function can be reduced to,

$J(\Theta, R)=\operatorname{det}(R)$.
Determination of the parameter vector $\Theta$ that minimizes $\operatorname{det}(\mathrm{R})$, or equivalently the function $J(\Theta, R)$, is an optimization problem that can be solved by applying different methods. The optimization algorithm can be summarized as follows (see also a scheme of the algorithm Fig. 10):

1) Choose suitable initial values for $\Theta$. With physical insight they can be around $50 \%$ of the real parameters;

2) Compute system outputs $y$ and the residuals $(z-a)$; estimate the measurement noise covariance matrix $R$;

3) Minimize $J(\Theta, R)$ with respect to $\Theta$ by applying one of the following optimization methods;

4) Iterate on step 2 and check for convergence. As we are dealing with a controlled system, inevitably the feedback will inject process noise in the system.

\section{Simulation results and parameter estimation}

As an example we have chosen the following parameter set to be estimated

$$
\begin{array}{r}
\Theta=\left[\begin{array}{lllllll}
a_{1} & a_{2} & a_{3} & a_{4} & a_{5} & a_{6} & K_{m 1} \\
K_{m 2} & \gamma_{11} & \gamma_{12} & K_{D F} & b_{x} & b_{y}
\end{array}\right]^{T}
\end{array}
$$

where

$a_{1}=\frac{\beta_{11}+\beta_{13}}{m_{1}}+\frac{\beta_{13}}{m_{2}}, \quad a_{2}=\frac{\beta_{12}+\beta_{13}}{m_{2}}+\frac{\beta_{13}}{m_{1}}$,

$a_{3}=\frac{k_{11}+k_{13}}{m_{1}}+\frac{k_{13}}{m_{2}}, \quad a_{4}=\frac{k_{12}+k_{13}}{m_{2}}+\frac{k_{13}}{m_{1}}$,

$a_{5}=\frac{\beta_{11}}{m_{1}}-\frac{\beta_{12}}{m_{2}}, \quad a_{6}=\frac{k_{11}}{m_{1}}-\frac{k_{12}}{m_{2}}$.

As mentioned before, when the coupling between the test masses is considered, the coefficients cannot be determined directly. They are calculated from the estimated parameters $\left[\begin{array}{llllll}a_{1} & a_{2} & a_{3} & a_{4} & a_{5} & a_{6}\end{array}\right]$. The state-space equations used are Eqs. (24) and (27). The test masses and the satellite were excited simultaneously with Multi-sines and Chirp excitation signals as shown in Table 1.

The Chirp signal was chosen to excite the test masses because the sinusoidal signal gives larger correlation between the estimated parameters and the PRBS cannot be used because of the controller structure.

The signals available for estimation were:

inputs $U_{1}, U_{2}, U_{S A T}, T_{1}, T_{2}$,

outputs $X_{1}, X_{2}, X_{\text {diff }}, \eta_{1}, \eta_{2}$

where $U_{1}, U_{2}, U_{S A T}$ are the inputs on the test mass 1,2 and satellite, respectively. $X_{1}$ and $X_{2}$ are the outputs of test mass 1

Table 1

Excitation signals used in the simulation

\begin{tabular}{lll}
\hline & Frequencies & Amplitude \\
\hline TM1/translation & 0.19 and $7 \mathrm{mHz}$ & $2 \times 10^{-10}$ \\
TM2/translation & 0.11 and $5 \mathrm{mHz}$ & $2 \times 10^{-10}$ \\
Torque around $\eta_{1}$ & $0.53 \mathrm{mHz}^{\mathrm{a}}$ & $2 \times 10^{-10}$ \\
Torque around $\eta_{2}$ & $1.10 \mathrm{mHz}^{\mathrm{a}}$ & $2 \times 10^{-10}$ \\
Satellite & $1.7-23 \mathrm{mHz}$ & $2 \times 10^{-7}$ \\
\hline
\end{tabular}

a Start frequency of a Chirp Signal. 

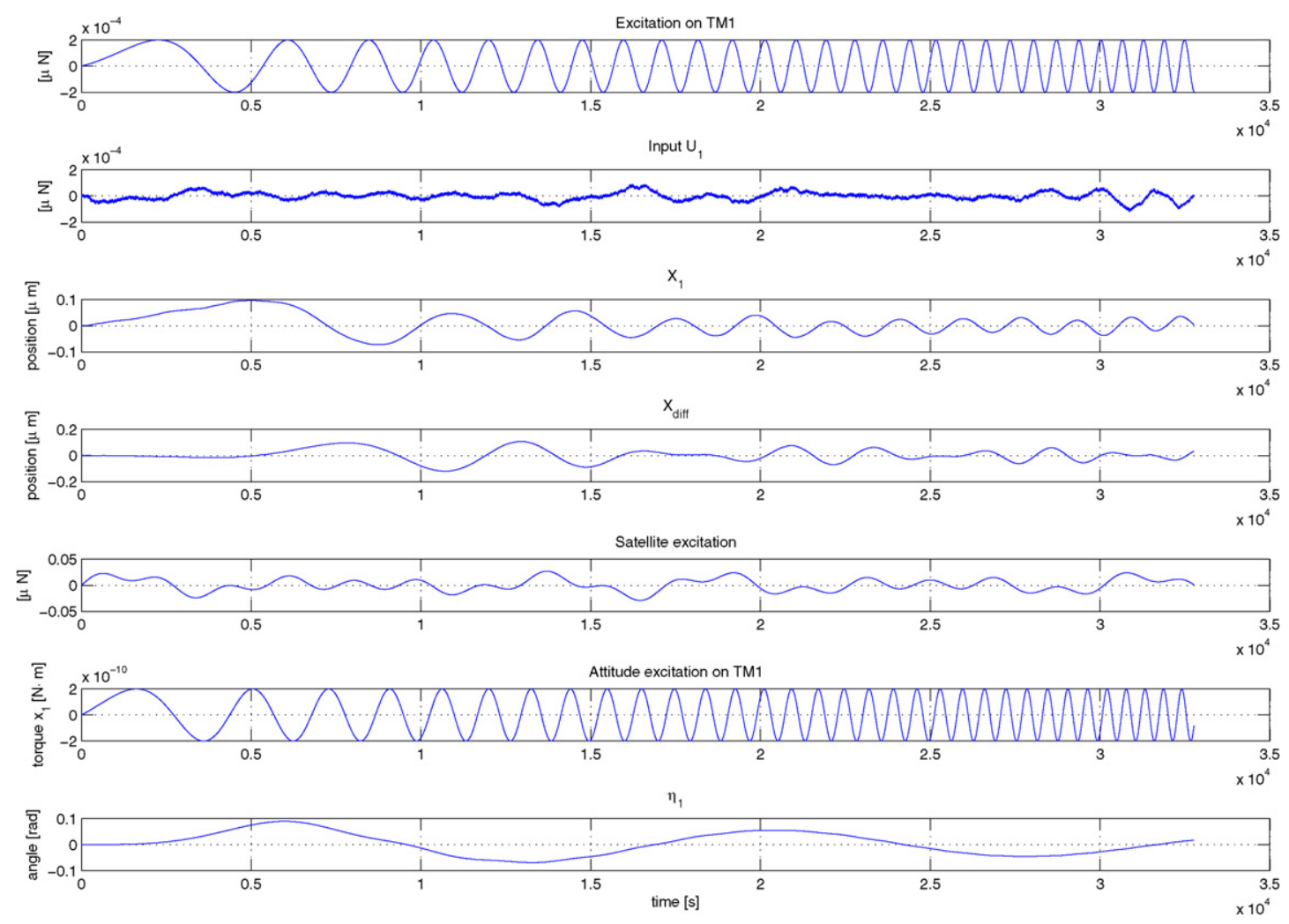

Fig. 11. Inputs and outputs used for parameter estimation. Simulation of a controlled Drag-Free Satellite with two test masses.

Table 2

Nominal and estimated parameters of a controlled system

\begin{tabular}{lllll}
\hline Parameter & Nominal value & OEM (error \%) & OLS (error \%) & TLS (error \%) \\
\hline$k_{11}$ & $1.482 \times 10^{-5}$ & $1.156 \times 10^{-5}(22.00)$ & $1.479 \times 10^{-5}(0.18)$ & $1.479 \times 10^{-5}(0.18)$ \\
$k_{12}$ & $2.223 \times 10^{-5}$ & $1.890 \times 10^{-5}(14.99)$ & $2.219 \times 10^{-5}(0.15)$ & $2.219 \times 10^{-5}(0.15)$ \\
$k_{13}$ & $3.947 \times 10^{-6}$ & $7.616 \times 10^{-6}(92.96)$ & $3.957 \times 10^{-6}(0.24)$ & $3.957 \times 10^{-6}(0.24)$ \\
$\beta_{11}$ & $1.3 \times 10^{-5}$ & $2.010 \times 10^{-5}(54.55)$ & $1.524 \times 10^{-5}(17.21)$ & $1.518 \times 10^{-5}(16.79)$ \\
$\beta_{12}$ & $1.95 \times 10^{-5}$ & $5.447 \times 10^{-5}(179.34)$ & $2.347 \times 10^{-5}(20.33)$ & $2.091 \times 10^{-5}(7.22)$ \\
$\beta_{13}$ & $6.93 \times 10^{-6}$ & $12.061 \times 10^{-6}(74.04)$ & $4.79 \times 10^{-6}(30.84)$ & $5.646 \times 10^{-6}(18.52)$ \\
$\gamma_{11}$ & $1.4 \times 10^{-11}$ & $1.398 \times 10^{-11}(0.14)$ & $1.4 \times 10^{-11}(0.00)$ & $1.401 \times 10^{-11}(0.07)$ \\
$\gamma_{12}$ & $1.0 \times 10^{-10}$ & $0.998 \times 10^{-11}(0.20)$ & $1.0 \times 10^{-11}(0.00)$ & $1.0 \times 10^{-11}(0.00)$ \\
$K_{m 1}$ & 1.0 & $0.999(0.00)$ & $1.0(0.00)$ & $1.0(0.00)$ \\
$K_{m 2}$ & 1.0 & $0.999(0.00)$ & $2.0 \times 10^{-3}(0.00)$ & $1.0(0.00)$ \\
$K_{D F}$ & $2.0 \times 10^{-3}$ & $2.0 \times 10^{-3}(0.00)$ & $0.424 \times 10^{-09}(57.60)$ & $1.987 \times 10^{-3}(0.65)$ \\
$b_{x}$ & $1.0 \times 10^{-09}$ & $1.627 \times 10^{-09}(37.30)$ & $0.57 \times 10^{-11}(43.00)$ & $1.58 \times 10^{-09}(58.00)$ \\
$b_{y}$ & $1.0 \times 10^{-11}$ & $1.501 \times 10^{-11}(49.90)$ & & $0.982 \times 10^{-11}(11.80)$ \\
\hline
\end{tabular}

and 2, respectively, and $X_{\text {diff }}$ the differential measurement (by SQUID or Laser Readout). $T_{1}$ and $T_{2}$ are the excitation torque on the test mass 1 and 2, respectively. $\eta_{1}$ and $\eta_{2}$ are the attitude angles coupled with the test masses displacement 1 and 2, respectively, in the sensitivity axis. It was assumed that the test body masses $m_{1}, m_{2}$ and $M$ are known.

Fig. 11 shows the excitation for test mass 1 and the measured input signal $U_{1}$ (excitation + control signal), along with the torque and the attitude angle for test mass 1 and the difference signal. Comparing the excitation on TM1 (first plot) and the input $U_{1}$ (second plot), one can see as the controller degrades the wave form of the signal. The input signal $U_{1}$ is very noisy (for $U_{2}$ input signal is the same). The excitation on the satellite is also shown. The test mass and satellite dynamic were simulated by using the Drag-Free Satellite Simulator built by ZARM [19]. Before the estimation parameter process takes place, the signals were filtered by a 5 th order Butterworth bandpass filter to exclude high frequency noise injected into the input by the controller. The estimated parameters are as shown in Table 2. It can be seen that the parameters are very well estimated.

\section{Conclusions}

This paper has shown a strategy to estimate/identify parameters of a drag-free satellite aiming to improve its control system. The system was simulated in a closed loop but the parameters were estimated in an "open-loop like" structure, i.e. taking only the plant input/output and not taking into consideration the con- 
troller structure. This is important because the drag-free attitude control system must be switched on all the time. It was shown also that any cross-coupling between the translational and rotational motions (cross-coupling or cross-talk) can be identified. LSE (OLS and TLS) and OEM + MLE methods were tested and the TLS method (with an ARX model) has shown better estimated parameters. The TLS method accounts for noise in the independent variables, i.e. in the regressors. However, the advantage of using the OEM method is the possibility to identify any systematic error in the input signals.

\section{References}

[1] P. Albertos, Iterative Identification and Control, Springer, 2002.

[2] D. Bortoluzzi, et al., Testing lisa drag-free control with the lisa technology package flight experiment, Classical and Quantum Gravity 20 (2003) S89S97.

[3] W. Fichter, P. Gath, S. Vitale, D. Bortoluzzi, Lisa pathfinder drag-free control and system implications, Classical and Quantum Gravity 22 (2005) 139-148.

[4] M.S. Guilherme, Electrostatic coupling between the test mass and the satellite for a cube accelerometer, Technical Report INT-DYN-TN-ZAR003, ZARM - University of Bremen, March 2006.

[5] E. Guiu, et al., Calibration methods for the test of the equivalence principle, in: Proceedings of the SYSID 2006, 4th IFAC Symposium on System Identification, January 2006

[6] V.M. Hannen, et al., End-to-end simulations for the lisa technology package, Classical and Quantum Gravity 20 (2003) S261-S271.

[7] R.V. Jategaonkar, Flight Vehicle System Identification, Progress in Astronautics and Aeronautics, vol. 216, American Institute of Aeronautics and Astronautics, Inc., 2006.
[8] H.P. Jin, Translation and attitude control for the QUICK-STEP satellite, $\mathrm{PhD}$ thesis, Department of Aeronautics and Astronautics of Stanford University, August 1996.

[9] S. Kirschstein, W. Alles, Identification of dynamic derivatives with controlled wind tunnel model of the spaceplane-configuration phoenix, Aerospace Science and Technology 8 (2004) 509-518.

[10] W.C. Leite Filho, M.S. Guilherme, S. Theil, In-orbit calibration of dragfree satellite dynamics, in: S.R. Vadali, et al. (Eds.), Spaceflight Mechanics 2006, Advances in the Astronautical Science, AAS/AIAA Space Flight Mechanics Meeting, Tampa, Florida, 2006, pp. 613-632.

[11] L. Ljung, System Identification: Theory for the User, second ed., Prentice Hall Information and Systems Sciences Series, Prentice Hall, 1999.

[12] S. Marcuccio, S. Giannelli, M. Andrenucci, Attitude and orbit control of small satellites and constellations with feep thrusters, in: IEPC-97-188, Proceedings of the 25th Electric Propulsion Conference, Cleveland, $\mathrm{OH}$, 1997.

[13] J. Mester, The step mission: principles and baseline design, Classical and Quantum Gravity 18 (2001) 2475-2486.

[14] K.S. Narendra, A.M. Annaswamy, Stable Adaptive Systems, PrenticeHall, 1989.

[15] G. Pradels, P. Touboul, In-orbit calibration approach of the microscope experiment for the test of the equivalence principle at $10^{-15}$, Classical and Quantum Gravity 20 (2003) 2677-2688.

[16] J. Schoukens, R. Pintelon, Identification of Linear Systems, A Practical Guideline to Accurate Modeling, Pergamon Press, 1991.

[17] T. Söderström, P. Stoica, System Identification, Prentice-Hall, 1989.

[18] STEP, Phase A Interim Report, Technical Report, NASA/ESA Joint STEP Working Group, 2001.

[19] S. Theil, Satellite and test mass dynamics modeling and observation for drag-free satellite control of the STEP mission, $\mathrm{PhD}$ thesis, Department of Production Engineering, University of Bremen, December 2002.

[20] P. Touboul, M. Rodrigues, The microscope space mission, Classical and Quantum Gravity 16 (2001) 2487-2498.

[21] B. Wie, Space Vehicle Dynamics and Control, AIAA Education Series, American Institute of Astronautics and Aeronautics, 1998. 